(CO), mixed venous saturation $\left(\mathrm{SvO}_{2}\right)$ and $\mathrm{PaCO}_{2}$; whereas significantly higher in pulmonary vascular resistance (PVR) and serum uric acid (UA) levels. Serum HDL-C levels positively correlated with $6 \mathrm{MWD}(\mathrm{r}=0.34, \mathrm{p}<0.001), \mathrm{CO}(\mathrm{r}=0.35, \mathrm{p}<0.001), \mathrm{SvO}_{2}(\mathrm{r}=0.40$, $\mathrm{p}<0.001)$ and $\mathrm{PaCO}_{2}(\mathrm{r}=0.289, \mathrm{p}<0.05)$; negatively correlated with UA levels $(r=-0.43, p<0.001)$ and PVR $(r=-0.30, p<0.05)$.

Conclusion Serum HDL-C levels correlated with the clinical severity of IPAH and maybe serve as a novel risk factor for the malignant disease.

\section{e0598 THE RESEARCH OF THE RELATION BETWEEN THE REFRACTORY HYPERTENSION AND THE HOMOCYSTEINE}

doi:10.1136/hrt.2010.208967.598

Yu Hao, Chen Su, Hu Xiaojun, Tu Yuanchao. Xinhua Hospital of Hubei Province

Objective To explore the relation between the refractory hypertension and the homocysteine (HCY).

Methods 164 patients with hypertension were randomly divided into refractory hypertension group $(n=76)$ and non-refractory hypertension group $(n=88)$, and meanwhile 60 healthy persons were regarded as the control group. we checked their homocysteine valuation and had the statistics analysis.

Results The homocysteine valuation of refractory hypertension group was significantly higher than those of the control group $(p<0.01)$ and was also higher than those of non-refractory hypertension group $(p<0.05)$. On the other hand. The homocysteine valuation of non-refractory hypertension group was higher than those of the control group $(\mathrm{p}<0.05)$.

Conclusion The blood homocysteine may be considered a relationship factor with refractory hypertension.

\section{e0599 THE IMPACT OF VALSARTAN ON ARTERIAL STIFFNESS IN PATIENTS WITH CORONARY HEART DISEASE AND HYPERTENSION}

doi:10.1136/hrt.2010.208967.599

Long Da, Liu Qiming. Department of Cardiology, the Second Xiangya Hospital of Central South University, Changsha

Objective To examine whether the angiotensin II receptor blockers valsartan would improve arterial stiffness to a greater extent than an equivalent antihypertensive medication, the calcium channel blocker amlodipine in patients with coronary heart disease combined with hypertension.

Methods 75 patients with coronary heart disease combined with hypertension in all accepted brachial-ankle pulse wave velelocity (ba-PWV) and central aortic blood pressure measurement. They were administered to take valsartan in a dose of $160 \mathrm{mg}$ per day (valsartan group, $\mathrm{n}=35$ ) or amlodipine $5-10 \mathrm{mg}$ per day (amlodipine group, $\mathrm{n}=40$ ) respectively soon after to ensure equivalent $\mathrm{BP}$ control. Measurements of ba-PWV and central aortic BP were carried out again after 24 weeks.

Results After 24 weeks there were no statistical differences in coronary artery Gensini score, left ventricular ejection fraction, mean heart rate and types of therapy medication between valsartan group and amlodipine group $(p>0.05)$. Systolic blood pressure, diastolic blood pressure and pulse blood pressure of brachial artery as well as central artery between baseline level and end of the study had no statistical differences between two groups ( $p>0.05$ ), and had significant decrease in both groups $(p<0.01)$. The levels of central artery BP and brachial BP controlled by valsartan were similar to amlodipine. There were no significant differences in ba-PWV between two groups at baseline (12.6 2.6 vs $2.1 \pm 2.6, p>0.05)$, however a significant decrease were observed in valsartan group after 24 weeks $(12.6 \pm 2.6$ vs $10.8 \pm 1.9, p<0.01)$, while no significant changes appeared in amlodipine group (12.1 22.6 vs $11.4 \pm 2.7$, $\mathrm{p}>0.05$ ).

Conclusion Valsartan may improve arterial stiffness to a significantly greater extent than amlodipine despite similar central artery and brachial BP control.

\section{E0600 IMPACTS OF OBSTRUCTIVE SLEEP APNOEA ON THE BLOOD PRESSURE IN HYPERTENSIVE PATIENTS UNDER THE OPTIMAL MEDICATION}

doi:10.1136/hrt.2010.208967.600

Zhao Qing, Liu Zhihong, Zhao Zhihui, Luo Qin, Zhang Hongliang, Wang Yong. Fuwai Hospital

Background and Objective Obstructive sleep apnoea (OSA) is a prevalent disease, however only $10 \%$ OSA patients receive regular treatment. OSA is an independent risk factor for hypertension and cardiovascular diseases. We aim to investigate the impacts of obstructive sleep apnoea on the blood pressure in hypertensive patients under the optimal medication though office blood pressure and $24 \mathrm{~h}$ ambulatory blood pressure monitoring ( $24 \mathrm{~h} \mathrm{ABPM}$ ), respectively.

Methods 52 patients with hypertension were enrolled consecutively and all received the optimal medication for hypertension. An overnight polysomnography and a $24 \mathrm{~h}$ ABPM were performed to each patient. According to the apnoea-hypopnoea index, the patients were divided into four groups: no OSA group ( $\mathrm{AHI}<5, \mathrm{n}=13$ ), mild OSA group $(5 \leq \mathrm{AHI}<15, \mathrm{n}=19)$, moderate $\mathrm{OSA}$ group $(15 \leq \mathrm{AHI}<30$, $n=11$ ), severe OSA group ( $A H I \geq 30, n=9)$. The results of $24 \mathrm{~h} A B P M$ and office pressure were compared respectively.

Results As to the $24 \mathrm{~h}$ ABPM results, $24 \mathrm{~h}$ systolic and diastolic pressures were significantly higher in severe OSA group than no OSA group ( $p$ value is 0.036 and 0.022 ), and night-time systolic and diastolic pressures were significantly higher too ( $p$ value is 0.046 and 0.024 ) in severe OSA group. Whereas no significant differences were found when compare day-time systolic and diastolic pressures between groups. Moreover, night-time diastolic pressure was significantly higher in severe OSA group than mild OSA group ( $p$ value is 0.039). After adjusting the confounders including age, sex, BMI, smoking and drinking history, and cardiovascular diseases, the statistic differences still remained. However, Office blood pressure including systolic and diastolic blood pressure had no significant differences between each two groups.

Conclusion Severe OSA significantly increases blood pressures, especially night-time blood pressures, of hypertensive patients who receive the optimal medication for hypertension. $24 \mathrm{~h} \mathrm{ABPM}$ is more accurate than office pressure to evaluate the blood pressure of hypertensive patients with OSA.

\section{e0601 EFFECTS OF BMI ON ISCHAEMIC STROKE IN HOSPITAL PATIENTS WITH ESSENTIAL HYPERTENSION}

doi:10.1136/hrt.2010.208967.601

${ }^{1}$ Yuexian Yao, ${ }^{1}$ Jinxiu Lin, ${ }^{2}$ Kaizu Xu. ${ }^{1}$ Department of Cardiology, The First Affiliated Hospital of Fujian Medical University, Fujian Hypertension Research Institute, ${ }^{2}$ Medical College of Putian University

Objective To investigate the association between BMI and the incidence of ischaemic stroke in essential hypertensive (EH) patients. 
Methods A total of $2158 \mathrm{EH}$ patients were divided into EH without ischaemic stroke group and $\mathrm{EH}$ with ischaemic stroke group. Four BMI quartiles were defined based on BMI level (The first quartile: BMI $\leq 22.68 \mathrm{~kg} / \mathrm{m}^{2}, \mathrm{n}=540$; The second quartile: BMI 22.69$24.82 \mathrm{~kg} / \mathrm{m}^{2}, \mathrm{n}=538$; The third quartile: BMI $24.83-26.84 \mathrm{~kg} / \mathrm{m}^{2}$, $\mathrm{n}=541$; The fourth quartile: $\mathrm{BMI} \geq 26.85 \mathrm{~kg} / \mathrm{m}^{2}, \mathrm{n}=539$ ). The incidence of ischaemic stroke in four BMI levels was evaluated by multivariable logistic regression analyses.

Results (1) The average BMI in EH with ischaemic stroke group is lower than that in $\mathrm{EH}$ without ischaemic stroke group (24.36 \pm 3.21 vs $25.15 \pm 3.31 \mathrm{~kg} / \mathrm{m}^{2}, \mathrm{p}<0.001$ ). (2) Form the first quartile to fourth quartile of BMI, the incidence of ischaemic stroke were decreasing $(37.6 \%, 34.8 \%, 30.9 \%, 23.0 \%, p<0.001)$. With $6.2 \%$ (95\% CI $3.3 \%$ to $9.0 \%$ ) decreased risk of incidence ischaemic stroke per $1 \mathrm{~kg} / \mathrm{m}^{2}$ increase of BMI. (3) Compared with the lowest BMI group, the adjusted $\mathrm{OR}(\mathrm{aOR})$ for ischaemic stroke in the highest BMI group was significantly lower (aOR: $0.800,95 \% \mathrm{CI}: 0.730$ to 0.875 , $\mathrm{p}<0.001)$. (4) In male group, comparing with the lowest BMI group, the aOR for ischaemic stroke in the highest BMI group was significantly lower (aOR: 0.811, 95\% CI: 0.717 to $0.918, p=0.001$ ); Similar trend was found in female group (aOR: 0.776, 95\% CI: 0.678 to $0.889, \mathrm{p}<0.001$ ).

Conclusion Lower BMI was associated with increased incidence of ischaemic stroke in $\mathrm{EH}$ patients.

\section{Clinical and Research Medicine: Organic Cardiovascular Disease (Myocarditis, CardioMyopathy, Congenital Heart Disease, Rheumatic Heart Disease, Valve e0602 THE PROGNOSIS INVESTIGATION IN PATIENTS WITH
CHRONIC HEART FAILURE AND PERICARDIAL EFFUSION}

doi:10.1136/hrt.2010.208967.602

Yu Shengbo, Zhao Qingyan, Huang He, Chen Donge, Cui Hongying, Huang Congxin. Renmin Hospital of Wuhan University

Background Researchers still do not reach the consensus on the incidence, characters and the prognostic value of pericardial effusion $(\mathrm{PE})$ in patients with chronic heart failure (CHF).

Methods 1189 patients, with a diagnosis of CHF consecutively admitted to three centers, were enrolled. M-mode echocardiography was used to determine the presence or absence of PE and to semiquantify it. 118 patients with PE and 472 without were followed up. The relationship between the $\mathrm{PE}$ and other parameters and the prognostic value of $\mathrm{PE}$ for $\mathrm{CHF}$ were analysed by univariate and multivariate analyses.

Results After follow up, 550 patients were analysed of which 226 were death. The incidence of PE was $9.92 \%$. Moderate PE was the most common which account $90.68 \%$ (107/118). 6.78\% (8/118) had small while only $2.54 \%(3 / 118)$ had large one. The systolic blood pressure $[\mathrm{OR}=1.04,95 \% \mathrm{CI}(1.01,1.07), \mathrm{p}=0.08]$, LVEF $[\mathrm{OR}=1.09$, $95 \% \mathrm{CI}(1.02,1.15), \mathrm{p}=0.06]$, and $\mathrm{MPAD}[\mathrm{OR}=1.51,95 \% \mathrm{CI}(1.24$, 1.85), $\mathrm{p}<0.001$ ] were the independent predictors of PE. The GFR $[\mathrm{OR}=1.013,95 \% \mathrm{CI}(1.005,1.026), \mathrm{p}=0.02]$, systolic blood pressure $[\mathrm{OR}=1.02,95 \% \mathrm{CI}(1.00,1.03), \mathrm{p}=0.015]$, LVEF $[\mathrm{OR}=1.08,95 \% \mathrm{CI}$ $(1.04,1.12), \mathrm{p}<0.001]$ and diabetes mellitus $[\mathrm{OR}=2.53,95 \% \mathrm{CI}$ $(0.99,6.44), \mathrm{p}<0.001]$ were determined as the independent predictors of CHF prognosis.

Conclusion The PE is not uncommon in CHF patients and most PE are small to moderate. PE is not related to the aetiology of $\mathrm{CHF}$ while is strongly connected with higher systolic blood pressure, lower LVEF and larger MPAD. PE dose not add the death risk of CHF patients.

\section{e0603 THE INHOSPITAL MORTALITY AND ITS DETERMINANTS FOR ELDERLY PATIENTS WITH ACUTE MYOCARDIAL INFARCTION}

doi:10.1136/hrt.2010.208967.603

Whenshu Zhao, Kuibao Li, Xinchun Yang. The heart center of Chaoyang hospital affiliated with Capital University of Medical Sciences

Objective To explore the inhospital mortality and its determinants for elderly patients with acute myocardial infarction.

Method Totally four hundreds and ninety nine patients with acute myocardial infarction, hospitalised in our hospital from January 1 , 2002 through February 22, 2010, who were equal or above 80 years older, were retrospectively analysed.

Results Among these 499 cases, 97 patients died during hospitalisation, with total inhospital mortality being $19.4 \%$. Of these patients, there were 297 cases with acute ST segment elevated myocardial infarction (STEAMI) and 202 cases with non -ST segment elevated myocardial infarction (NSTEAMI), and their inhospital mortality were $23.2 \%$ and $13.9 \%$, respectively $(p=0.009$ ). Univariable analysis showed the proportions of infection, digest tract bleeding, history of hypertension, complete atrial-ventricular (A-V) block, cardiac rupture, three or four killip grade of cardiac function, and STEAMI in death group were higher than that in alive group $(p<0.05)$, and the mortality of those who did not undergo urgent PCI was higher $(p<0.05)$. Multivariable logistic regression analysis showed the independent determinants for mortality of elderly AMI patients were cardiac killip grades, complete A-V block, renal dysfunction, stent implant, and the type of AMI.

Conclusions The independent determinants for mortality of elderly AMI patients are as following, cardiac killip grade, complete A-V block, renal dysfunction, stent implant, and the type of MAI. Urgent PCI is safe and effective for elderly with AMI.

\section{E0604 MRI CHARACTERISTICS : COMPARISON OF ARRHYTHMOGENIC RIGHT VENTRICULAR CARDIOMYOPATHY PATIENTS WITH AND WITHOUT SYNCOPE}

doi:10.1136/hrt.2010.208967.604

Cheng Huaibing, Zhao Shihua, Jiang Shiliang, Lu Minjie, Ling Jian, Zhan Yan, Yan Chaowu, Liu Oiong, Ma Ning, Li Shiguo, Yin Gang. Department of Radiology, Cardiovascular Institute and Fuwai Hospital, Chinese Academy of Medical Sciences and Peking Union Medical College

Objective This study was designed to review MRI characteristics and assessed its risk factors for life-threatening ventricular arrhythmia in arrhythmogenic right ventricular cardiomyopathy (ARVC)

Methods We collected a consecutive series of 63 patients with clinical diagnosis of ARVC at a single institution. In all cases the diagnosis was performed according to ESC/ISFC diagnostic criteria. MRI characteristics were compared between patients with syncope and concomitantly sustained ventricular tachycardia or ventricular fibrillation (group 1) and remaining patients (group 2). Morphological and functional parameters and tissue differentiation were assessed.

Results Univariate analysis showed significantly differences between both groups in terms of familial history of ARVC or sudden death $(14 \%$ vs $41 \%, p=0.015)$, the accordion sign $(58 \%$ vs $81 \%$, $\mathrm{p}=0.031$ ), left ventricular (LV) involvement ( $47 \%$ vs $74 \%, p=0.032$ ), number of regions with intramyocardial fat infiltration $(2.4 \pm 1.4 \mathrm{vs}$ $3.1 \pm 1.5, \mathrm{p}=0.047)$, number of regions with myocardial fibrosis $(1.0 \pm 0.9$ vs $1.6 \pm 0.9, p=0.013)$. No differences were noted when comparing baseline characteristics of the patient population. A binary logistic regression model showed that familial history of 